\title{
Sind die Thromben anders als bei Nichtdiabetikern?
}

Fragestellung: Gibt es bei Diabetikern Besonderheiten bei Koronarplaques und -thromben, die ihre Gefährlichkeit erklären?

Hintergrund: Akute koronare Ischämien sind bei Diabetikern häufig und besonders gefürchtet. Sie entstehen aus atherosklerotischen Plaques, die bei Diabetikern eine dünnere fibröse Kappe aufweisen, stärker zur Ruptur neigen und einen größeren Lipidkern enthalten. Der Myokardinfarkt tritt ein, wenn der Plaque erodiert und rupturiert mit gleichzeitiger Thrombosebildung. Das erhöhte Risiko für kardiovaskuläre Komplikationen von Diabetikern im Vergleich zu Nichtdiabetikern könnte auf besonderen Eigenschaften des Thrombus beruhen.

Patienten und Methoden: Zwischen April 2010 und Dezember 2012 erhielten Patienten mit akutem Infarkt eine perkutane Koronarintervention. Gleichzeitig wurde Thrombusmaterial aspiriert und anschließend morphologisch und histopathologisch untersucht, um Hinweise auf die Pathophysiologie des Infarktgeschehens zu erhal-

\section{Originalie}

Sebben J C, Ribeiro D R P, Lopes $R D$ et al. The role of diabetes mellitus in the composition of coronary thrombi in patients presenting with acute ST-segment elevation myocardial infarction undergoing primary percutaneous coronary intervention. Am Heart J 2016;172:26-33. ten. Dabei wurden alle mikro- und makroskopischen Analysen von drei Pathologen durchgeführt, die keine Kenntnis über den Diabetesstatus oder andere Patientendaten hatten.

Ergebnisse: Unter den 259 Patienten waren 49 (19\%) Diabetiker und 210 Nichtdiabetiker, die - ausgenommen

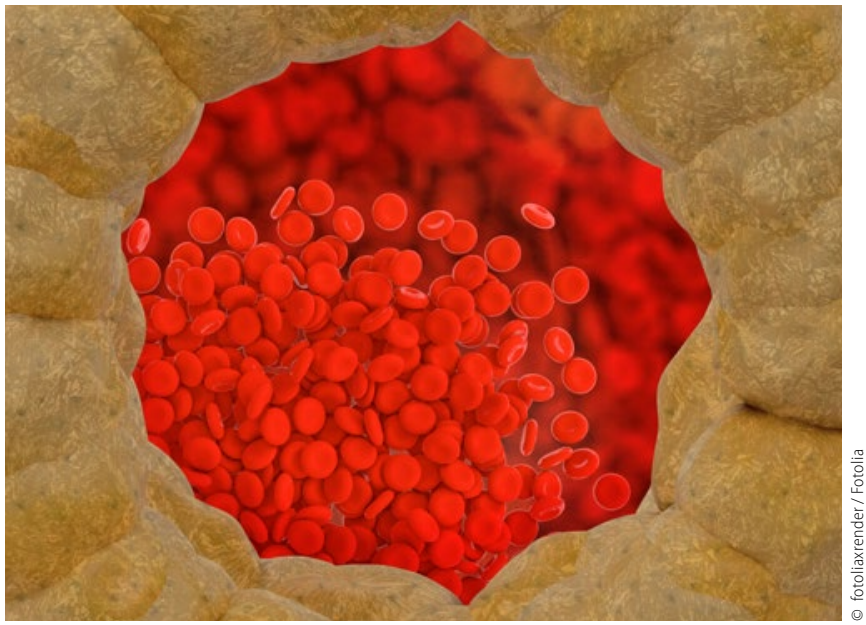

Ob und inwieweit Blut von Diabetespatienten anders verklumpt als das Blut Stoffwechselgesunder, das konnte diese Studie nicht klären.

Antidiabetika - die gleiche medikamentöse Therapie erhielten. Die Thromben waren makroskopisch nach Größe, Farbe, Alter, Volumen und Zahl der Fragmente, mikroskopisch in der $\mathrm{Zu}$ sammensetzung von Leukozyten, Thrombozyten und Fibrin in beiden Gruppen nicht unterschiedlich. Das gleiche gilt auch für Todesfälle und schwere kardiovaskuläre Komplikationen in den ersten 30 Tagen nach dem Infarkt.

Schlussfolgerung: In der Zusammensetzung der Thromben nach akutem Herzinfarkt gab es bei Diabetikern und Nichtdiabetikern keine Unterschiede.

\section{- Kommentar von Prof. Dr. med. Heinrich Holzgreve}

\section{Komplexität der Biologie hält Frage offen}

Verschiedene Publikationen beschreiben eine erhöhte Thrombozytenaktivität und eine stärkere Koagulationsneigung bei Diabetikern. Tatsächlich wurden auch Unterschiede der Koronarthromben bei Diabetikern und Nichtdiabetikern beschrieben, allerdings bei postmortal gewonnenem Material. Einerseits wird dieser Mangel bei der Thrombusaspiration beim frischen Infarkt vermieden und ermöglicht die Untersuchung eines höchst komplexen biologischen Prozesses in vivo. Andererseits kann die in der Regel vor Beginn der Katheteruntersuchung einsetzende pharmakologische Lyse das Thrombusbild verfälschen. Leider bleibt also offen, ob sich die Thrombusqualität bei Diabetikern und Nichtdiabetikern unterscheidet und ob sich daraus Konsequenzen für die thrombolytische Therapie ableiten lassen.

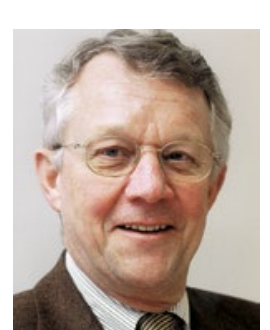

Prof. Dr. med. Heinrich Holzgreve

Internist, Kardiologische Praxis

Burgstr. 7, 80331 München

h.holzgreve@t-online.de 